\title{
Nucleon mass and isovector couplings in $2+1$-flavor dynamical domain-wall lattice QCD near physical mass
}

\author{
Michael Abramczyk $\odot,{ }^{1}$ Thomas Blum, ${ }^{1,2}$ Taku Izubuchi (出渕卓), ${ }^{3,2}$ Chulwoo Jung, ${ }^{3,2}$ Meifeng Lin, ${ }^{4}$ \\ Andrew Lytle $\odot,{ }^{5}$ Shigemi Ohta (太田滋生), ${ }^{6,7,2}$ and Eigo Shintani (新谷栄悟) ${ }^{8}$ \\ (RBC and UKQCD Collaborations) \\ ${ }^{1}$ Physics Department, University of Connecticut, Storrs, Connecticut 06269-3046, USA \\ ${ }^{2}$ RIKEN-BNL Research Center, Brookhaven National Laboratory, Upton, New York 11973, USA \\ ${ }^{3}$ Physics Department, Brookhaven National Laboratory, Upton, New York 11973, USA \\ ${ }^{4}$ Computational Science Initiative, Brookhaven National Laboratory, Upton, New York 11973, USA \\ ${ }^{5}$ INFN, Sezione di Roma Tor Vergata, Via della Ricerca Scientifica 1, 00133 Roma RM, Italy \\ ${ }^{6}$ Theory Center, The High Energy Accelerator Research Organization (KEK), \\ Oho 1-1, Tsukuba, Ibaraki 3050801, Japan \\ ${ }^{7}$ Deaprtment of Particle and Nuclear Physics, The Graduate University of Advanced Studies \\ (SOKENDAI), Hayama, Kanagawa 2400193, Japan \\ ${ }^{8}$ RIKEN Advanced Institute of Computational Sciences (AICS), Kobe, Hyogo 650-0047, Japan
}

(Received 8 November 2019; accepted 21 January 2020; published 13 February 2020)

\begin{abstract}
We report nucleon mass, isovector vector and axial-vector charges, and tensor and scalar couplings, calculated using two recent $2+1$-flavor dynamical domain-wall fermion lattice-QCD ensembles generated jointly by the RIKEN-BNL-Columbia and UKQCD Collaborations. These ensembles were generated with Iwasaki $\times$ dislocation-suppressing-determinant-ratio gauge action at an inverse lattice spacing of 1.378(7) $\mathrm{GeV}$ and pion mass values of 249.4(3) and 172.3(3) MeV. The nucleon mass extrapolates to a value $m_{N}=$ $0.950(5) \mathrm{GeV}$ at the physical point. The isovector vector charge renormalizes to unity in the chiral limit, narrowly constraining excited-state contamination in the calculation. The ratio of the isovector axial-vector to the vector charges shows a deficit of about $10 \%$. The tensor coupling no longer depends on mass and extrapolates to 1.04(5) in $\overline{\mathrm{MS}} 2 \mathrm{GeV}$ renormalization at the physical point, in a good agreement with the value obtained at the lightest mass in our previous calculations and other calculations that followed. The scalar charge, though noisier, does not show mass dependence and is in agreement with other calculations.
\end{abstract}

DOI: 10.1103/PhysRevD.101.034510

\section{INTRODUCTION}

The RIKEN-BNL-Columbia (RBC) Collaboration and subsequently the joint RBC and UKQCD Collaborations have been investigating nucleon structure using the domain-wall fermion (DWF) quarks on a sequence of quenched [1,2], two-flavor [3], and 2+1-flavor [4-6] dynamical DWF ensembles at various mass values [7-12]. As is well known, the DWF scheme allows us to maintain continuum-like flavor and chiral symmetries on the lattice, and it helps to simplify nonperturbative renormalizations [13-16]. In this paper, we report nucleon isovector vector

Published by the American Physical Society under the terms of the Creative Commons Attribution 4.0 International license. Further distribution of this work must maintain attribution to the author(s) and the published article's title, journal citation, and DOI. Funded by SCOAP ${ }^{3}$. and axial-vector charges, and tensor $\left(g_{T}^{u-d}=\langle 1\rangle_{\delta u-\delta d}\right)$ and scalar $\left(g_{S}^{u-d}\right)$ couplings calculated using two recent $2+1$-flavor dynamical DWF lattice-QCD ensembles generated jointly by the RBC and UKQCD Collaborations with Iwasaki $\times$ dislocation-suppressing-determinant-ratio (DSDR) gauge action at $\beta=1.75, a^{-1}=1.378(7) \mathrm{GeV}$, and pion masses of 249.4(3) and 172.3(3) MeV [11,12], which are significantly lighter than in our previous nucleon works.

In our earlier works, calculated with degenerate up- and down-quark masses set to be considerably heavier than physical values [4-6], we observed the vector-current form factors behaving reasonably well in trending toward experiments: both Dirac and Pauli mean-squared charge radii and the isovector anomalous magnetic moment appeared to linearly depend on the pion mass squared. The radii extrapolated to the physical mass undershoot the experimental value by about $25 \%[17,18]$. It would have been 
interesting to see if the present calculation confirmed this earlier trend, or if it could help resolve the discrepancy seen between the muon Lamb shift experiment [17] and older electron scattering ones. However, our current numerical precision from relatively small statistics and large momentum transfer is yet to be competitive with the Lamb shift experiments $[17,18]$ which now are prevailing $[19,20]$, so we would like to defer reporting our form factors at finite momentum transfers until a future date when we will have better precision.

In our earlier calculation of axial-vector current form factors, we saw a significant deficit in the calculated axialvector charge, $g_{A}$, and form factors in general appear more susceptible to finite-size effects than the vector-current ones $[4,5]$. We find in our present calculations at lighter pion mass that this deficit persists, and we investigate potential causes for this in detail in Sec. V.

In contrast, the isovector tensor coupling showed an interesting downward departure at the lightest mass to about 1.0, away from the flat higher-mass values of about 1.1 [6]. Whether this trending continues in our present calculations at considerably lighter mass is obviously an interesting question. The tensor and scalar couplings are also relevant to the search for new physics beyond the standard model such as the neutron electric dipole moment $[21,22]$.

Some preliminary analyses of these nucleon observables have been reported at recent lattice conferences [23-27]. In addition, the LHP Collaboration also calculated some nucleon observables [28] using a RBC + UKQCD 2 + 1flavor dynamical DWF ensemble [9].

\section{NUMERICAL METHOD}

In this paper, we concentrate on our results for mass and four isovector couplings of nucleons: vector and axialvector charges and tensor and scalar couplings. Though we summarize their definitions and computational methods later in this section, we refer the readers to our earlier publication [5] for the full details.

The two $2+1$-flavor dynamical domain-wall fermion gauge field ensembles we use in this work [11,12] were generated jointly by the RBC and UKQCD Collaborations on a $32^{3} \times 64$ four-dimensional volume and $L_{s}=32$ in the fifth dimension with Iwasaki $\times$ DSDR gauge action at the gauge coupling of $\beta=1.75$ and $2+1$-flavors of dynamical DWF quarks with a domain-wall height of 1.8, strange-quark mass of 0.045 , and degenerate up- and down-quark masses of 0.0042 and 0.001 in lattice units. These parameters result in an inverse lattice spacing, $a^{-1}$, of $1.378(7) \mathrm{GeV}$ and a DWF residual quark mass of $0.001842(7)$. Note that the inverse lattice spacing has been slightly revised from the original $[10,11]$, using the global chiral and continuum fits in conjunction with new physical-mass ensemble sets [12] with Möbius DWF quarks. Thus, the heavier of the two ensembles corresponds to a pion mass $m_{\pi}$ of 249.4(3) MeV and a spatial lattice extent $L$ of $m_{\pi} L=5.79(6)$, and the lighter to 172.3(3) $\mathrm{MeV}$ and 4.00(6), respectively. Our measurement calculations were made using 165 configurations between the molecular dynamics (MD) trajectories 608 and 1920 with an 8-trajectory interval for the former, and using 39 configurations between 748 and 1420 with a 16-trajectory interval for the latter.

We refer to our earlier publications $[3,4]$ for the details of two- and three-point correlation functions for the nucleon. A conventional nucleon operator, $N(x)=$ $\epsilon_{a b c}\left\{\left[u_{a}(x)\right]^{T} C \gamma_{5} d_{b}(x)\right\} u_{c}(x)$, with color indices $a, b$, and $c$, quark flavors $u$ for up and $d$ for down, and charge conjugation operator $C=\gamma_{4} \gamma_{2}$ is used. Additionally, gauge-invariant Gaussian smearing $[29,30]$ is applied to suppress excited-state contamination: For both ensembles, we compared the Gaussian widths of 4 and 6 lattice units and found that the wider width- 6 results settle on a plateau more quickly, and the narrower width-4 results merge with them. From these observations, we concluded that the wider width-6 Gaussian smearing is sufficient for our study.

Isospin symmetry is enforced for the up and down quarks, and we calculate only proton matrix elements of the third isospin component of the vector and axial-vector currents:

$$
\begin{array}{r}
\left\langle P\left|V_{\mu}^{3}(x)\right| P\right\rangle=\left\langle P\left|\bar{u}(x) \gamma_{\mu} u(x)-\bar{d}(x) \gamma_{\mu} d(x)\right| P\right\rangle, \\
\left\langle P\left|A_{\mu}^{3}(x)\right| P\right\rangle=\left\langle P\left|\bar{u}(x) \gamma_{5} \gamma_{\mu} u(x)-\bar{d}(x) \gamma_{5} \gamma_{\mu} d(x)\right| P\right\rangle .
\end{array}
$$

All quark-disconnected diagrams cancel in these matrix elements.

We use a source-sink separation of 9 lattice units, or about $1.3 \mathrm{fm}$. This is sufficiently long for the observables reported in this paper to be free of excited-state contamination, as is demonstrated below with augmentative calculations with a source-sink separation of 7 lattice units $[24,25]$. For the two-point correlation functions we use the same Gaussian-smeared sources and point or Gaussiansmeared sinks: We will refer to the former as G-L and the latter as G-G, respectively. We also calculate the tensor coupling, $g_{T}=\langle 1\rangle_{\delta q}$, and scalar coupling, $g_{S}$, the same way. We use a conventional measurement strategy for the former with seven source-sink pairs for each configuration, and an "all-mode averaging" (AMA) strategy [31] for the latter with 112 sloppy solves with sources at two sets of eight evenly spaced spatial locations, from $(0,0,0)$ to $(16$, $16,16)$ and from $(8,8,8)$ to $(24,24,24)$ for $T=0,8,16,24$, 32,40 , and 48 , and four precise solves at spatial origins with $T=0,16,32$, and 48 for each configuration. In addition, to test for excited-state contamination, calculations with a source-sink separation of 7 lattice units were made with 64 sloppy solves with sources at eight evenly spaced spatial locations with $T=0,8,16,24,32,40,48$, and 56, and one precise solve at spatial origins with $T=0$. 


\section{NUCLEON MASS}

Table I summarizes the nucleon energies obtained from correlated, single-exponential fits to the G-G two-point correlation function. To improve statistics, the correlation function is averaged over forward- and backward-propagating nucleon and antinucleon states. The fit ranges were chosen after inspecting the effective masses and taking the shortest distance from the source consistent with an acceptable $\chi^{2}$ value from the fit. Increasing this minimum distance by a single time unit does not change the energy beyond the statistical error. From these we estimate the nucleon mass for the present ensembles as summarized in Table II and Fig. 1. Whereas our prior calculations at heavier pion masses found nucleon masses extrapolating to values much higher than experiment, pion masses now are sufficiently close to physical values that the new data extrapolates, linearly in terms of pion mass squared $\left(m_{\pi}^{2}\right)$, to a value $m_{N}=0.6894(7) a^{-1}=0.6894(7) \times$ $1.378(7) \mathrm{GeV}=0.950(5) \mathrm{GeV}$. This extrapolation is only slightly more than 2 standard deviations away from the average of the proton and neutron masses, 0.938918747(6) GeV [19]. The slope in this linear extrapolation is steeper than that observed in our earlier calculations with heavier mass: the result constrains nonlinear dependence of nucleon mass on pion mass squared.

TABLE I. Fitted nucleon energy obtained from the Gaussiansmeared sink two-point correlation function $(\mathrm{G}-\mathrm{G})$.

\begin{tabular}{lcccc}
\hline \hline$m m_{q} a$ & $|\vec{p}|^{2}$ & Fit range & Energy & $\chi^{2} /$ d.o.f. \\
\hline 0.001 & 0 & $5-10$ & $0.707(5)$ & 0.33 \\
0.001 & 1 & $5-10$ & $0.734(5)$ & 0.13 \\
0.001 & 2 & $5-10$ & $0.761(6)$ & 0.16 \\
0.001 & 3 & $5-10$ & $0.786(6)$ & 0.10 \\
0.001 & 4 & $5-10$ & $0.807(8)$ & 0.72 \\
0.0042 & 0 & $6-12$ & $0.765(12)$ & 1.55 \\
0.0042 & 1 & $6-12$ & $0.790(13)$ & 1.62 \\
0.0042 & 2 & $6-12$ & $0.816(14)$ & 2.01 \\
0.0042 & 3 & $6-12$ & $0.84(2)$ & 2.20 \\
0.0042 & 4 & $6-12$ & $0.86(2)$ & 1.77 \\
\hline \hline
\end{tabular}

TABLE II. Nucleon mass estimates. We also list the revised numbers for the two lightest ensembles of Refs. [5,6] with the new and more accurate estimate for the inverse lattice spacing [12].

\begin{tabular}{llll}
\hline \hline$a^{-1}[\mathrm{GeV}]$ & $m_{q} a$ & \multicolumn{1}{c}{$m_{N} a$} & $m_{N}[\mathrm{GeV}]$ \\
\hline $1.378(7)$ & 0.001 & $0.7077(8)$ & $0.9752(11)$ \\
& 0.0042 & $0.7656(2)$ & $1.0550(3)$ \\
$1.7848(5)$ & 0.005 & $0.6570(9)$ & $1.1726(16)$ \\
& 0.01 & $0.7099(5)$ & $1.2670(9)$ \\
\hline \hline
\end{tabular}

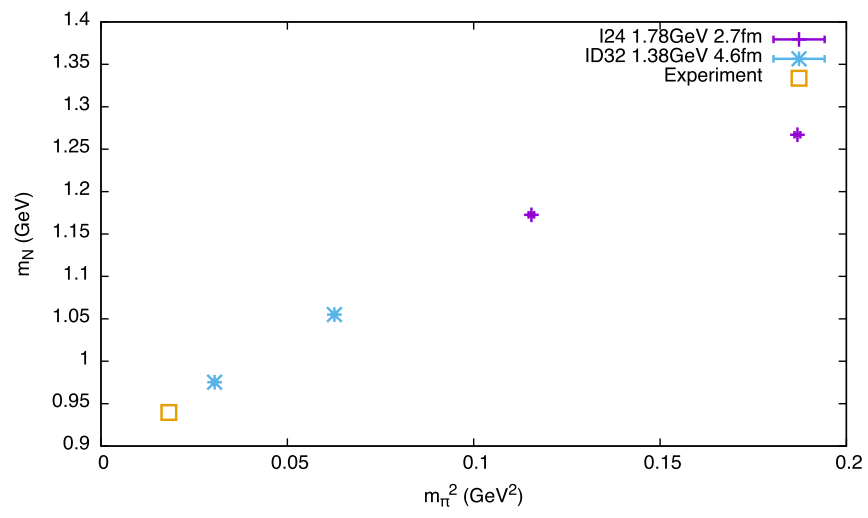

FIG. 1. Estimated nucleon mass, $m_{N}$, plotted against estimated pion mass squared, $m_{\pi}^{2}$, of the present ensembles (ID32, cyan) and the two lightest of Refs. [5,6] (I24, magenta) with the new and more accurate estimate for the inverse lattice spacing [12]. The present results linearly extrapolate to the experiment $(\square)$ within the statistical error.

\section{VECTOR CHARGE}

Signals for the isovector vector charge, $g_{V}$, are shown in Fig. 2 for both the $m_{\pi}=172.3(3)$ and 249.4(3) MeV ensembles. Robust time-independent plateaus are seen. In the following, we average values in the range $3 \leq t_{\mathrm{op}} \leq 6$, and find 1.450(4) for the heavier and 1.447(9) for the lighter ensemble, respectively. The values compare with the inverse of the vector-current renormalization, $Z_{V}$, computed in the meson sector [11], 0.664(5) $)^{-1}=1.506(11)$ and $0.669(4)^{-1}=1.495(9)$. Alternatively, we linearly extrapolate the two calculated vector-charge values of $1.450(4)$ at $m_{f} a=0.0042$ and $1.447(9)$ at 0.001 to the chiral limit, $m_{f} a=-m_{\text {residual }} a=-0.0018427$, to obtain a value 1.474(11). This is to be compared with the inverse of the meson-sector vector-current renormalization, $Z_{V}$, in the chiral limit, $Z_{V}^{-1}=0.673(8)^{-1}=1.49(2)$. Thus, the

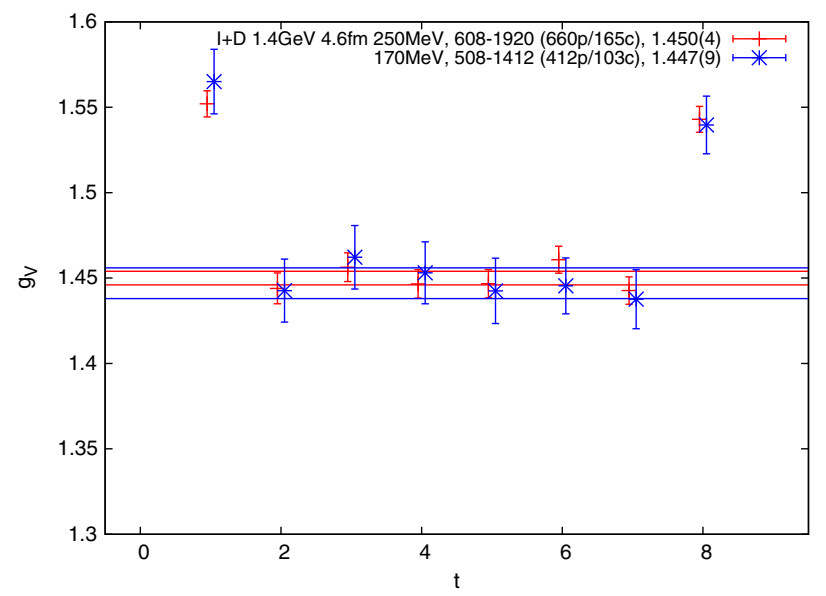

FIG. 2. Isovector vector charge, $g_{V}$, from $m_{\pi}=172.3(3)$ and 249.4(3) MeV ensembles. 


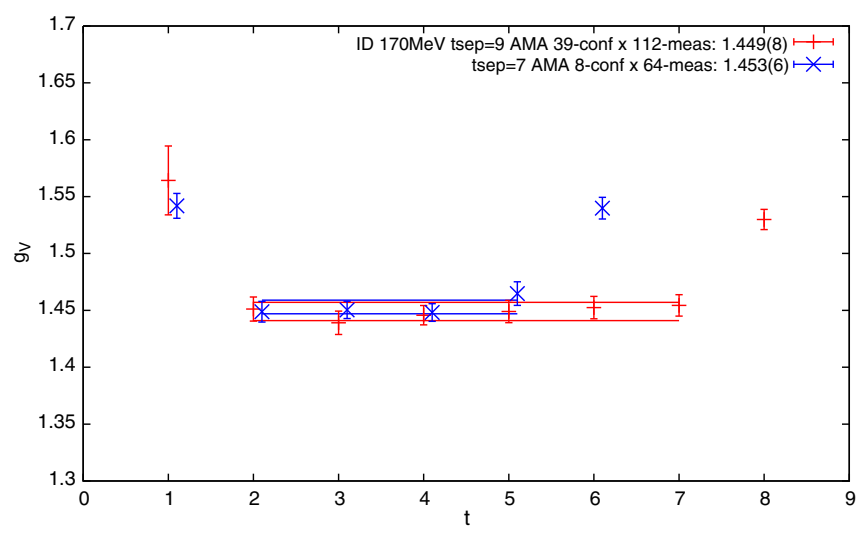

FIG. 3. Signals for isovector vector charge $g_{V}$, from source-sink separations of 7 and 9 lattice units, agree to within 0.6 percent, well within statistical errors.

nucleon vector charge in the chiral limit renormalizes to unity within statistical errors: $Z_{V} g_{V}=0.992(14)$.

Had the renormalized vector charge $Z_{V} g_{V}$ deviated from unity, a likely cause would be excited-state contamination, $\sum_{\text {excited states }}\left\langle\right.$ excited state $\left.\left|g_{V}\right| 0\right\rangle$, through violation of vector-current conservation at $O\left(a^{2}\right)$ in lattice spacing $a$. That our vector charge renormalizes to unity within a couple of percent statistical error constrains such excited-state contamination. As is shown in Fig. 3, signals for isovector vector charge $g_{V}$, from source-sink separations of 7 and 9 lattice units, agree to within 0.6 percent, well within statistical errors. If we assume the first excited state is the ground-state nucleon plus a pion of mass $m_{\pi} a=0.1249$ (2) with a unit of lattice momentum $2 \pi / L=0.1963$, then it decays as $\exp (-2 \times(0.1249+0.1963))=0.5260$ in 2 lattice units of time from 7 to 9 . So the relative amplitude of this state in the source times the $O\left(a^{2}\right)$ mixing matrix element cannot exceed approximately $1 \%$. It may appear that the statistics used for this comparison is small, but it is sufficient for confirming that the results from the short and long source-sink separations are in agreement with each other. This is because the short- and long-separation results are correlated within each configuration. Indeed such a correlation can be directly studied by limiting the comparison to configurations between trajectories 748 and 908 : there are all eight configurations for separation 7 , and an additional three for 9 . The jackknife difference between the two separations is consistent with zero except for separation 6, where a larger DWF fifth-dimensional effect is expected in the separation 7 result (see Fig. 4.) Hence, we conclude that there is no evidence for excited-state contamination detected in the most precise of our observables, the vector charge, in the lighter ensemble. In addition, the source smearing study performed for both ensembles did not suggest any different behavior in the heavier ensemble, and the lighter ensemble should have a denser excitation spectrum. Accordingly, possible excited-state contamination in the heavier ensemble must be smaller than in the

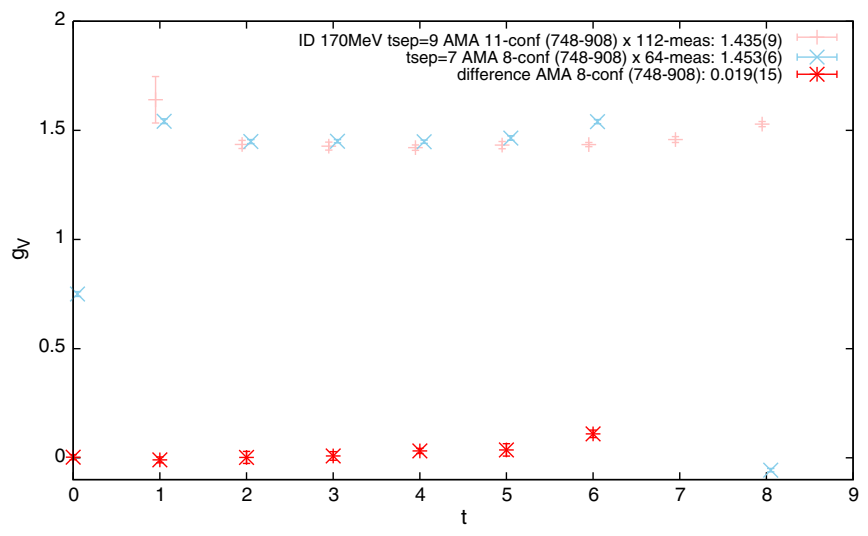

FIG. 4. Direct comparison of signals for isovector vector charge $g_{V}$ from source-sink separations of 7 and 9 lattice units agree for trajectories 748, 764, 796, 828, 844, 876, 892, and 908 for 7 units and for three more in the same range for 9 units. The difference is consistent with zero, except for separation 6, where a larger DWF fifth-dimensional effect can be seen for separation 7 .

lighter, as the excitations are farther away. There is no evidence for excited-state contamination in our ensembles.

\section{AXIAL-VECTOR CHARGE}

As is the case for the vector current, we use the localcurrent definition for the axial-vector current. Because the two local currents are connected by a chiral rotation, they share a common renormalization, $Z_{A}=Z_{V}$, that relates them with the corresponding conserved global currents, up to $O\left(a^{2}\right)$ discretization. This is an advantage of the DWF scheme. Thus, for the axial-vector charge $g_{A}$, it is better to look at its ratio $g_{A} / g_{V}$ to the vector charge, for precision, as is demonstrated in Fig. 5. The calculated value of the ratio $g_{A} / g_{V}$ underestimates the experimental value of 1.2732(23) [19] by about $10 \%$ and does not depend much on the pion mass, $m_{\pi}$, in the range from about $418.8(1.2) \mathrm{MeV}$ down to 172.3(3) MeV from four recent RBC + UKQCD 2+1flavor dynamical DWF ensembles [9-11] (see Fig. 6.). The result appears to have been confirmed by several other major collaborations [21,32-35] using different actions but with similar lattice spacings and quark masses, though extrapolations to physical mass seem to differ. It is especially important for calculations with Wilson-fermion quarks [21,32] to remove the $O(a)$ systematic errors at the linear order in the lattice spacing $a$ [33].

We are obviously suffering from some systematics that make our calculations undershoot the experimental value of $g_{A} / g_{V}=1.2732(23)$ [19]. Indeed, we see possible signs of inefficient sampling: First, we observe an unusually longrange autocorrelation when we divide the lightest ensemble at $m_{\pi}=172.3$ (3) $\mathrm{MeV}$ into two halves, earlier and later, in hybrid MD time, as in Fig. 7. Indeed, when we further divide the ensemble into four consecutive quarters in MD time, the axial-vector charge starts at a value consistent 

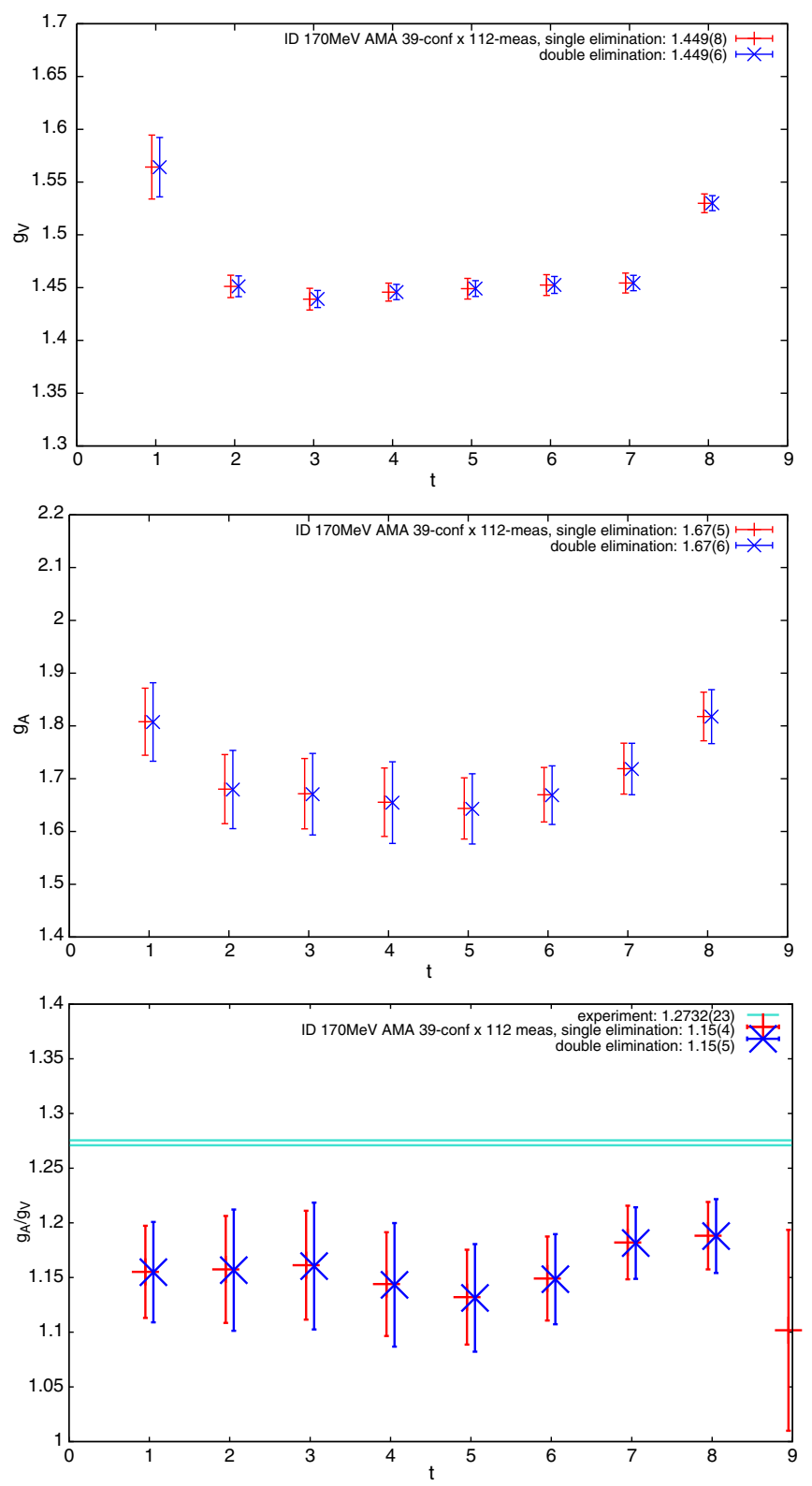

FIG. 5. Comparative signal qualities of the isovector vector, $g_{V}$, and axial, $g_{A}$, charges and their ratio, $g_{A} / g_{V}$, from the $172 \mathrm{MeV}$ ensemble. The charges are bare values from the local currents, and the ratio is naturally renormalized, as the two currents share the common renormalization. Clearly, the ratio is more precisely determined than the axial-vector charge.

with experiment but monotonically decreases to a value below unity, as in Fig. 8.

Importantly, we also note that no such undersampling is seen in any other isovector observables we have looked at, including the vector charge $g_{V}$, the quark momentum fraction $\langle x\rangle_{u-d}$, and the quark helicity fraction $\langle x\rangle_{\Delta u-\Delta d}$, and that blocked jackknife analyses with block sizes of 2 and 3 show a strong correlation of two successive gauge configurations for $g_{A}$ and $g_{A} / g_{V}$. Somewhat weaker autocorrelations may be seen in some other observables to a block size of 2 , but then they disappear at block size 3 .

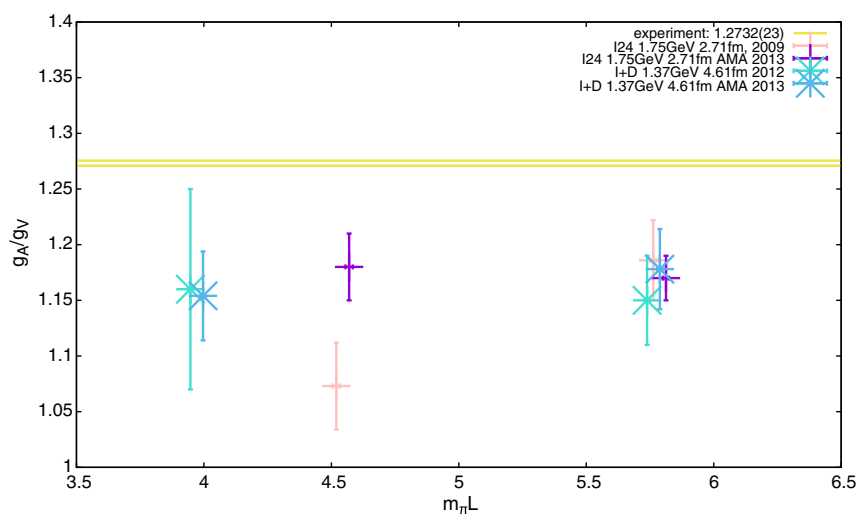

FIG. 6. Comparison of the dependence of the ratio, $g_{A} / g_{V}$, of isovector axial-vector charge $g_{A}$ and vector charge $g_{V}$, calculated with recent $\mathrm{RBC}+\mathrm{UKQCD} 2+1$-flavor dynamical DWF ensembles, on the finite-size scaling parameter, $m_{\pi} L$, in the present paper and our earlier reports. The experimental value quoted here is 1.2732(23), from the latest PDG [19].

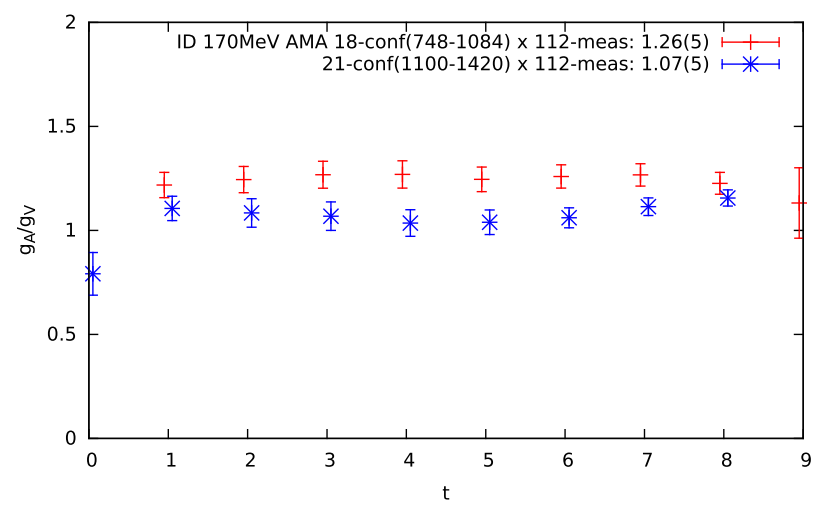

FIG. 7. Plateaus of the ratio $g_{A} / g_{V}$ for the first (trajectory from 748 to 1084 , red) and the second (1100 to 1420 , blue) halves, respectively: fitted in the range from 2 to 7 lattice units, the values of 1.26(5) for the first half and 1.07(5) for the second half are almost 4 standard deviations away from each other.

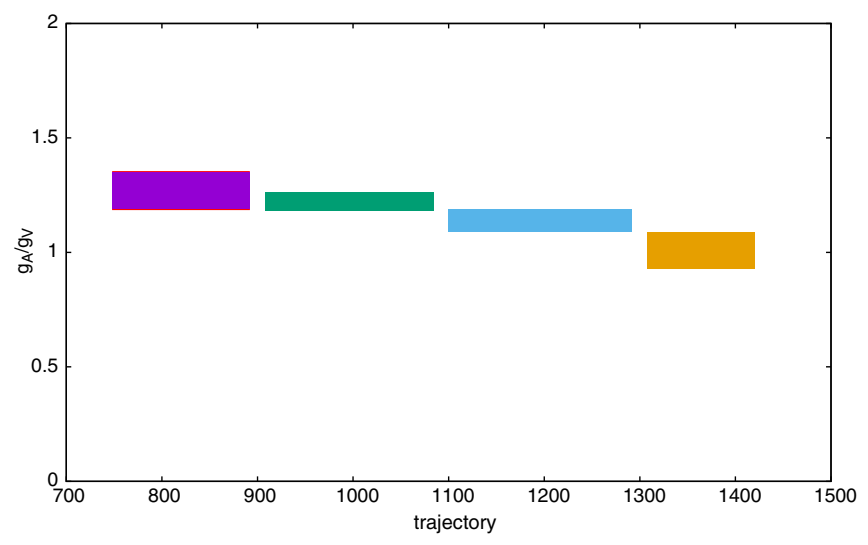

FIG. 8. Quarterwise average along the hybrid MD time, from 748 to 892,908 to 1084,1100 to 1292 , and 1308 to 1420 : The values drift monotonically from what is consistent with experiment in the first quarter to a value around 1.0 in the last quarter. 


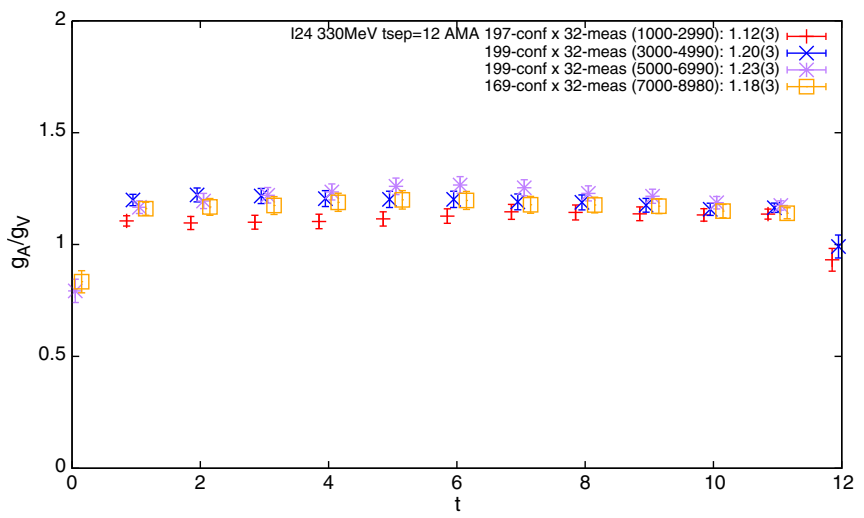

FIG. 9. A weaker but similar long-range autocorrelation is also seen in $g_{A} / g_{V}$ in the $m_{\pi}=331.3(1.4) \mathrm{MeV}$ ensemble when it is divided into quarters.

A similar but weaker sign of unusually long-range autocorrelation can be seen in the lightest of our earlier ensembles [9] at $m_{\pi}=331.3$ (1.4) $\mathrm{MeV}$ when we divide it into four consecutive quarters in hybrid MD time, as in Fig. 9. However, no such sign of undersampling is seen in the other two ensembles - the present one at $m_{\pi}=249.4(3) \mathrm{MeV}$, or another at 418.8(1.2) MeV from the earlier work [9]. In other words, the strongest sign of undersampling is seen at the smallest finite-size scaling parameter, $m_{\pi} L \sim 4.00(6)$. Another weaker indication of a long-range autocorrelation effect is seen at the second smallest parameter, but not the second lightest, at $m_{\pi} L \sim 4.569(15)$. No effect is seen for larger values at $m_{\pi} L \sim 5.79(6)$ or 5.813(12). This, of course, does not prove that the problem is caused by the finite lattice spatial volume, but it suggests so.

That there is a long-range autocorrelation in this observable is corroborated by blocked jackknife analysis with block sizes of 2, 3, and 4, as is summarized in Table III: The statistical error of the axial-vector charge keeps growing to at least beyond a block size of 3 , while those for the other observables do not grow at all, except perhaps for tensor coupling, which nonetheless stops growing earlier.

TABLE III. Summary of blocked jackknife analysis for some isovector observables. The error does not grow with block size except for the axial-vector charge and tensor coupling.

\begin{tabular}{|c|c|c|c|c|}
\hline \multicolumn{5}{|c|}{ Blocked jackknife analysis } \\
\hline & \multicolumn{4}{|c|}{ Block size } \\
\hline & 1 & 2 & 3 & 4 \\
\hline$g_{V}$ & $1.447(8)$ & $1.447(6)$ & $\cdots$ & $\ldots$ \\
\hline$g_{A}$ & $1.66(6)$ & $1.66(7)$ & $1.66(8)$ & $1.65(4)$ \\
\hline$g_{A} / g_{V}$ & $1.15(4)$ & $1.15(5)$ & $1.15(6)$ & $1.14(3)$ \\
\hline$\langle x\rangle_{u-d}$ & $0.146(7)$ & $0.146(8)$ & $0.146(8)$ & $\ldots$ \\
\hline$\langle x\rangle_{\Delta u-\Delta d}$ & $0.165(9)$ & $0.165(11)$ & $0.165(10)$ & $\ldots$ \\
\hline$\langle x\rangle_{u-d}^{\Delta u-\Delta a} /\langle x\rangle_{\Delta u-\Delta d}$ & $0.86(5)$ & $0.86(4)$ & $\ldots$ & $\ldots$ \\
\hline$\langle 1\rangle_{\delta u-\delta d}$ & $1.42(4)$ & $1.42(6)$ & $1.42(6)$ & $1.41(3)$ \\
\hline
\end{tabular}

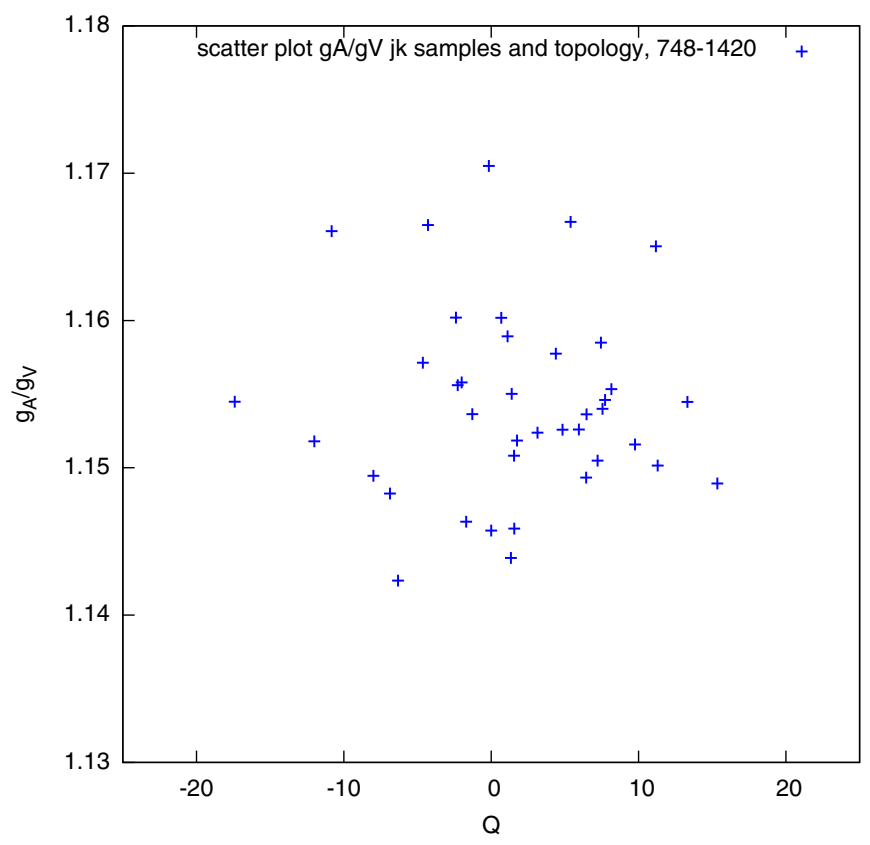

FIG. 10. Scatter plot of $g_{A} / g_{V}$ jackknife samples against gauge topological charge: No correlation is seen.

If an observable appears to have long-range autocorrelation, it would be interesting to look at its correlation with the topology of the gauge configurations. We explored this possibility by plotting jackknife samples against the topological charge (see Fig. 10), and we did not find a correlation.

We can also look at whether our low-mode deflation affected this, though the available information is limited to about half of the configurations of what we are presenting from the $172 \mathrm{MeV}$ ensemble (see Fig. 11.) Albeit with this limitation, we do not find any correlation either: The average of the lowest 100 eigenmodes does not differ between the two halves. Some difference emerges as we

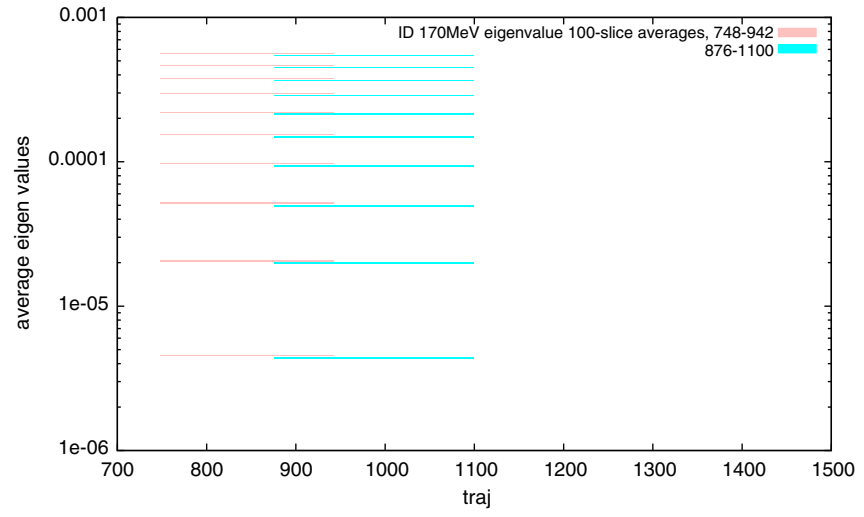

FIG. 11. Deflation eigenmode statistics for the two quarters where data are available: There is no difference in the lowest 100 modes averaged. Some insignificant difference emerges in higher modes. 

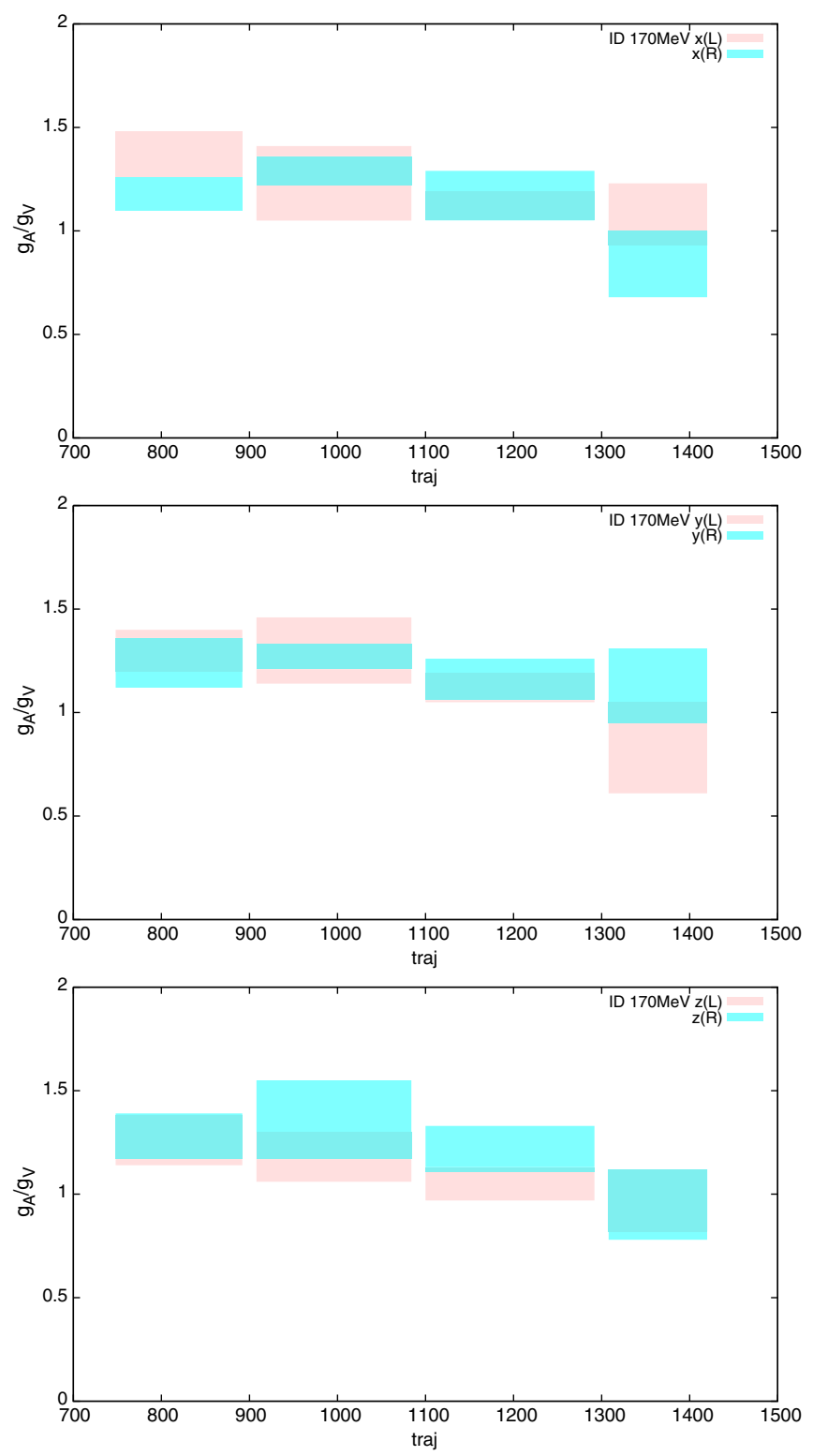

FIG. 12. Evolution of the ratio $g_{A} / g_{V}$ along the course of molecular dynamics time, divided into two spatial halves in the $x$ (top), $y$ (middle), and $z$ (bottom) directions. The calculation appears to fluctuate spatially. Spin polarization is along the $z$ axis.

go to higher eigenmodes, but it does not appear to be significant.

On the other hand, as pointed out earlier in this paper, similar long-range autocorrelation was seen in the 331.3 (1.4) MeVensemble [24] that is at the second smallest $m_{\pi} L$, but not in the lighter 249.4(3) MeV nor the heaviest 418.8 (1.2) $\mathrm{MeV}$ ensembles with larger $m_{\pi} L$, hinting that the systematics may arise from the finite-size effect.

It may be also instructive to remember earlier phenomenological analyses such as one performed using the MIT bag model that estimates $g_{A} / g_{V}=1.09$ without the pion [36], and another by the Skyrmion model that gives only a conditionally convergent result of 0.61 that is strongly dependent on pion geometry [37].

To explore such spatial dependence arising from pion geometry, we divided the AMA samples into two spatial halves such as $0 \leq x<L / 2$ and $L / 2 \leq x<L$ for each of the three spatial directions in order to check if there is any uneven spatial distribution (see Fig. 12.) We found that the calculation fluctuates in space. A larger spatial volume would stabilize the calculation better.

\section{TENSOR AND SCALAR COUPLINGS}

Plateau signals for the bare isovector tensor, $g_{T}=$ $\langle 1\rangle_{\delta u-\delta d}$, and the scalar coupling, $g_{S}$, are presented in Fig. 13. The tensor-coupling signals are very clean and do not show any mass dependence. As was mentioned earlier, this observable shows weaker but still relevant signs of long-lasting autocorrelation similar to that of the axialvector charge in the lighter 172.3(3) $\mathrm{MeV}$ ensemble [25]. Yet the agreement with the heavier ensemble, where there is no such autocorrelation, reassures that this is less problematic in the tensor coupling than in the axial-vector charge. The scalar plateaus are also well defined, albeit with larger statistical errors. No mass dependence can be seen here either. In addition, the flatness of the plateaus within respective statistical errors for both observables
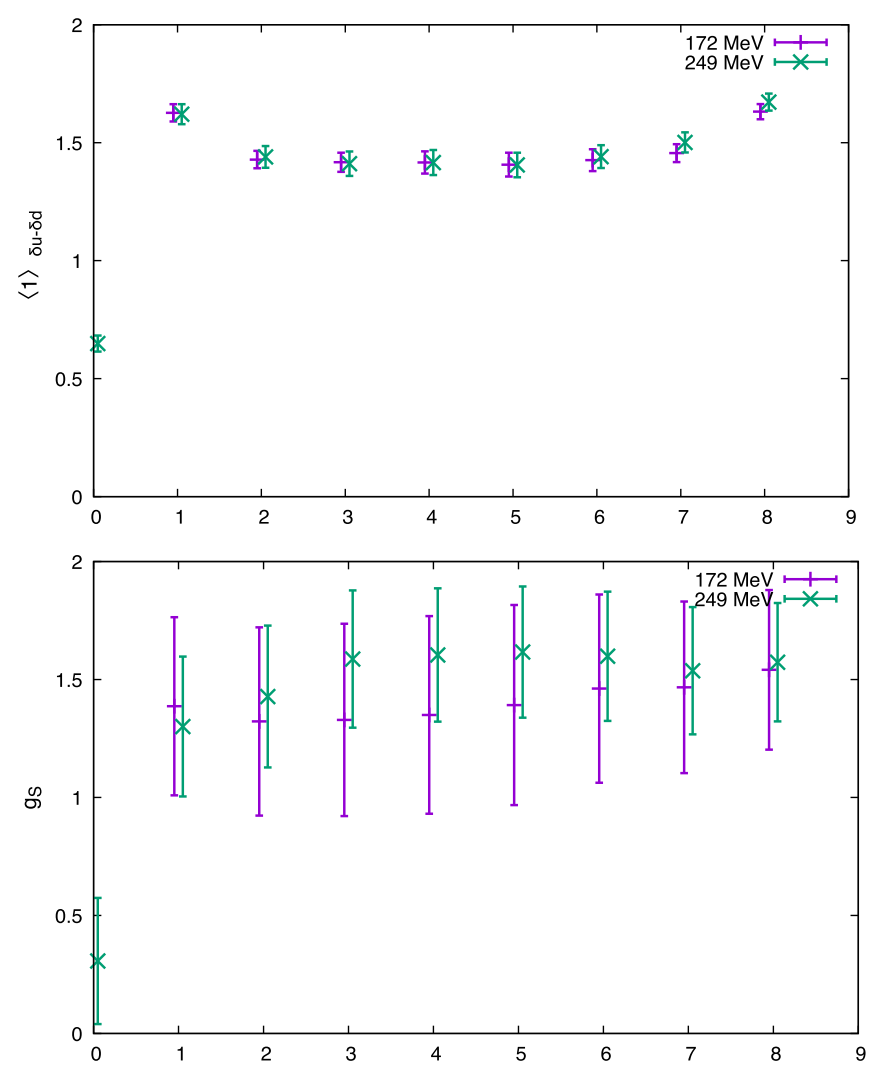

FIG. 13. Bare isovector tensor, $g_{T}=\langle 1\rangle_{\delta u-\delta d}$, and scalar, $g_{S}$, coupling plateau signals. The horizontal axes are lattice time in lattice units. 
does not indicate the measurable presence of excited-state contamination.

Bilinear operators are renormalized using the RomeSouthampton nonperturbative renormalization (NPR) method [13]. This method allows the determination of lattice matching factors ( $Z$ factors) to regularization-independent (RI) schemes using lattice simulations directly, and without recourse to lattice perturbation theory. The validity of the method requires that the renormalization scale $\mu$ be sufficiently separated from the QCD scale and the scale of the lattice cutoff:

$$
\Lambda_{\mathrm{QCD}} \ll \mu \ll \frac{\pi}{a}
$$

A perturbative calculation in the continuum is then conventionally used to convert the RI-scheme results to the $\overline{\mathrm{MS}}$ scheme.

The relation between bare (lattice) operators and their renormalized counterparts is

$$
\psi_{\mathrm{R}}=Z_{q}^{1 / 2} \psi_{\mathrm{B}}, \quad \mathcal{O}_{\mathrm{R}}=Z_{\mathcal{O}} \mathcal{O}_{\mathrm{B}} .
$$

Here $\mathcal{O}$ is one of $S, V$, or $T$ for the scalar, vector, and tensor bilinears, respectively. We compute the amputated Landaugauge fixed Green's functions of the operators of interest between external quark states with the momenta $p_{1}$ and $p_{2}$. Let these quantities be denoted $\Lambda_{\Gamma}\left(p_{1}, p_{2}\right)$, where $\Gamma$ is one of $\left\{S=1, V=\gamma^{\mu}, T=\sigma^{\mu \nu}\right\}$. Here we use the RI/SMOM scheme defined in Ref. [16], for which the momenta satisfy $p_{1}^{2}=p_{2}^{2}=\left(p_{1}-p_{2}\right)^{2}$. This differs from the original RI/ MOM scheme proposal [13], which instead takes $p_{1}=p_{2}$. This improved kinematic setup maintains a single renormalization scale, but it ensures momentum flows through the vertex, thereby suppressing unwanted infrared effects. It was also found in Ref. [16] that the RI/SMOM-to- $\overline{\mathrm{MS}}$ matching factors have smaller $\mathcal{O}\left(\alpha_{s}\right)$ coefficients.

There are two principal SMOM variants defined in Ref. [16], called RI/SMOM and RI/SMOM ${ }_{\gamma_{\mu}}$, which differ in their definitions of wave-function renormalization. The bilinear $Z$ factors are determined at the scale $|p|$ by imposing the conditions

$$
\begin{aligned}
\frac{1}{12} \operatorname{Tr}\left[\Lambda_{S, \mathrm{R}}\left(p_{1}, p_{2}\right)\right] & =1, & & \\
\frac{1}{144} \operatorname{Tr}\left[\sigma_{\mu \nu} \Lambda_{T, \mathrm{R}}^{\mu \nu}\left(p_{1}, p_{2}\right)\right] & =1, & & \\
\frac{1}{12 q^{2}} \operatorname{Tr}\left[q_{\mu} \Lambda_{V, \mathrm{R}}^{\mu}\left(p_{1}, p_{2}\right) q\right] & =1, & & (\mathrm{RI} / \mathrm{SMOM}) \\
\frac{1}{48} \operatorname{Tr}\left[\gamma_{\mu} \Lambda_{V, \mathrm{R}}^{\mu}\left(p_{1}, p_{2}\right)\right] & =1, & & \left(\mathrm{RI} / \mathrm{SMOM}_{\gamma_{\mu}}\right)
\end{aligned}
$$

on the amputated Green's functions in the chiral limit. Here the traces are over both spin and color indices, and the normalization factors are such that Eq. (5) is satisfied by the bare operators in the free theory.

The propagators used to construct $\Lambda_{\Gamma}\left(p_{1}, p_{2}\right)$ are computed using momentum sources, which results in very low statistical noise using a modest number of configurations. Additionally, twisted boundary conditions on the quark fields allow us to continuously vary the magnitude of $p$ while keeping the orientation fixed, resulting in smooth data as a function of the renormalization scale.

Results for the RI/SMOM and RI/SMOM $\gamma_{\mu}$ intermediate schemes are presented in Tables IV-VI. The wave-function renormalization factors have been removed using $Z_{\mathcal{V}} / Z_{q}$ determined from Eq. (5), where $Z_{\mathcal{V}}$ relates the local 4D current to the conserved 5D current. These results are then converted to the $\overline{\mathrm{MS}}$ scheme using the two-loop perturbative expressions calculated in Refs. [38,39].

TABLE IV. Scalar and tensor renormalization constants in the RI/SMOM $\left(\gamma_{\mu}\right)$ schemes at $m_{\pi}=172.3(3) \mathrm{MeV}_{\text {. }}$

\begin{tabular}{lccccccc}
\hline \hline$\mu[\mathrm{GeV}]$ & 1.53 & 1.63 & 1.72 & 1.82 & 1.91 & 2.00 & 2.11 \\
\hline$Z_{\mathrm{S}} / Z_{\mathcal{V}}\left(\mathrm{RI} / \mathrm{SMOM}_{\gamma_{\mu}}\right)$ & $0.813(1)$ & $0.829(2)$ & $0.842(2)$ & $0.855(1)$ & $0.866(1)$ & $0.878(1)$ & $0.888(1)$ \\
$Z_{\mathrm{S}} / Z_{\mathcal{V}}(\mathrm{RI} / \mathrm{SMOM})$ & $0.892(1)$ & $0.906(2)$ & $0.918(2)$ & $0.930(1)$ & $0.940(1)$ & $0.951(1)$ & $0.960(1)$ \\
$Z_{\mathrm{T}} / Z_{\mathcal{V}}\left(\mathrm{RI} / \mathrm{SMOM}_{\gamma_{\mu}}\right)$ & $1.073(1)$ & $1.066(1)$ & $1.060(1)$ & $1.054(1)$ & $1.049(1)$ & $1.045(1)$ & $1.041(1)$ \\
$Z_{\mathrm{T}} / Z_{\mathcal{V}}(\mathrm{RI} / \mathrm{SMOM})$ & $1.177(1)$ & $1.166(1)$ & $1.155(1)$ & $1.146(1)$ & $1.138(1)$ & $1.131(1)$ & $1.125(1)$ \\
\hline \hline
\end{tabular}

TABLE V. Scalar and tensor renormalization constants in the RI/SMOM$\left(\gamma_{\mu}\right)$ schemes at $m_{\pi}=249.4(3) \mathrm{MeV}$.

\begin{tabular}{lccccccc}
\hline \hline$\mu[\mathrm{GeV}]$ & 1.53 & 1.63 & 1.72 & 1.82 & 1.91 & 2.00 & 2.11 \\
\hline$Z_{\mathrm{S}} / Z_{\mathcal{V}}\left(\mathrm{RI} / \mathrm{SMOM}_{\gamma_{\mu}}\right)$ & $0.814(1)$ & $0.829(1)$ & $0.842(1)$ & $0.855(1)$ & $0.866(1)$ & $0.877(1)$ & $0.887(1)$ \\
$Z_{\mathrm{S}} / Z_{\mathcal{V}}(\mathrm{RI} / \mathrm{SMOM})$ & $0.894(1)$ & $0.907(1)$ & $0.918(1)$ & $0.930(1)$ & $0.940(1)$ & $0.950(1)$ & $0.959(1)$ \\
$Z_{\mathrm{T}} / Z_{\mathcal{V}}\left(\mathrm{RI} / \mathrm{SMOM}_{\gamma_{\mu}}\right)$ & $1.073(1)$ & $1.066(1)$ & $1.060(1)$ & $1.054(1)$ & $1.049(1)$ & $1.045(1)$ & $1.041(1)$ \\
$Z_{\mathrm{T}} / Z_{\mathcal{V}}(\mathrm{RI} / \mathrm{SMOM})$ & $1.178(1)$ & $1.166(1)$ & $1.156(1)$ & $1.146(1)$ & $1.138(1)$ & $1.132(1)$ & $1.126(1)$ \\
\hline \hline
\end{tabular}


TABLE VI. Scalar and tensor renormalization constants in the RI/SMOM$\left(\gamma_{\mu}\right)$ schemes in the chiral limit.

\begin{tabular}{lccccccc}
\hline \hline$\mu[\mathrm{GeV}]$ & 1.53 & 1.63 & 1.72 & 1.82 & 1.91 & 2.00 & 2.11 \\
\hline$Z_{\mathrm{S}}\left(\mathrm{RI} / \mathrm{SMOM}_{\gamma_{\mu}}\right)$ & $0.546(8)$ & $0.557(9)$ & $0.566(9)$ & $0.575(8)$ & $0.583(8)$ & $0.591(8)$ & $0.598(8)$ \\
$Z_{\mathrm{S}}(\mathrm{RI} / \mathrm{SMOM})$ & $0.599(8)$ & $0.610(9)$ & $0.617(9)$ & $0.626(8)$ & $0.632(8)$ & $0.640(8)$ & $0.646(8)$ \\
$Z_{\mathrm{T}}\left(\mathrm{RI} / \mathrm{SMOM}_{\gamma_{\mu}}\right)$ & $0.722(8)$ & $0.717(8)$ & $0.713(8)$ & $0.709(8)$ & $0.706(8)$ & $0.703(8)$ & $0.700(8)$ \\
$Z_{\mathrm{T}}(\mathrm{RI} / \mathrm{SMOM})$ & $0.792(8)$ & $0.785(8)$ & $0.777(8)$ & $0.771(8)$ & $0.766(8)$ & $0.761(8)$ & $0.757(8)$ \\
\hline \hline
\end{tabular}

TABLE VII. Renormalized isovector tensor $\left(g_{T}=\langle 1\rangle_{\delta u-\delta d}\right)$ and scalar $\left(g_{S}\right)$ couplings.

\begin{tabular}{lcc}
\hline \hline$m_{\pi}[\mathrm{MeV}]$ & $g_{T}$ & $g_{S}$ \\
\hline $172.3(3)$ & $1.04(5)$ & $0.9(3)$ \\
$249.4(3)$ & $1.04(5)$ & $1.0(2)$ \\
\hline \hline
\end{tabular}

Taking the average of results from the two intermediate schemes after conversion to modified MS, and taking the full difference as an estimate of the systematic, we find for the renormalization factors of the scalar and tensor couplings $Z_{S}(\overline{\mathrm{MS}}, 2 \mathrm{GeV})=0.642(8)(22)$ and $Z_{T}(\overline{\mathrm{MS}}, 2 \mathrm{GeV})=0.731(8)(24)$.

From these, we obtain our estimates for the renormalized isovector tensor and scalar couplings, as presented in Table VII. Neither is dependent on mass.

The tensor coupling is in good agreement with a value, about 1.0, obtained at the lightest mass in our previous calculations [6], and also with later calculations by others [40,41]. Its errors are dominated by scheme-dependent systematics in nonperturbative renormalization, at about $5 \%$, due mainly to the relatively low lattice cutoff.

The scalar coupling, though noisier, is in broad agreement with other later calculations $[40,41]$. The scalar errors are still dominated by statistical noise, but they will eventually encounter the same scheme-dependent systematics in nonperturbative renormalization.

\section{CONCLUSIONS}

The nucleon masses calculated in the two present ensembles extrapolate linearly in pion mass squared, $m_{\pi}^{2}$, to a value $m_{N}=0.950(5) \mathrm{GeV}$ at the physical point. This is to be compared with the average of the proton and neutron experimental mass values, 0.938918747(6) GeV [19]. The slope in this linear extrapolation is steeper than that observed in our earlier calculations with heavier mass - the result constrains the nonlinear dependence of nucleon mass on the pion mass squared.

The isovector vector charge renormalizes to unity in the chiral limit. This narrowly constrains the excited-state contamination in the Gaussian smearing.

The ratio of the isovector axial-vector to the vector charge shows a deficit of about $10 \%$. This is in agreement with some other major lattice numerical calculations [21,32-35] using different actions but with similar lattice spacings and quark masses. The origin of this deficit is still to be understood.

We obtained good signals for the isovector tensor coupling. It does not depend on mass and extrapolates to 1.04(5) at physical mass with the $\overline{\mathrm{MS}} 2 \mathrm{GeV}$ renormalization. This is in agreement with the value obtained for the lightest pion mass of 331.3(1.4) MeV in our earlier work [6], and also with calculations by others [40,41].

The isovector scalar coupling is noisier, but again it does not show mass dependence, and it is in agreement with other calculations $[40,41]$.

\section{ACKNOWLEDGMENTS}

The ensembles were generated using four QCDOC computers of Columbia University, Ediburgh University, the RIKEN-BNL Research Center (RBRC), and USQCD Collaboration at Brookhaven National Laboratory, and a Bluegene/P computer of the Argonne Leadership Class Facility (ALCF) of the Argonne National Laboratory provided under the INCITE Program of the U.S. Department of Energy. Calculations of nucleon observables were done using the RIKEN Integrated Cluster of Clusters (RICC) at RIKEN, Wako, and various Teragrid and XSEDE clusters of the U.S. National Science Foundation. S. O. was partially supported by the Japan Society for the Promotion of Sciences under Kakenhi Grant No. 15K05064.
[1] S. Sasaki, K. Orginos, S. Ohta, and T. Blum (RIKEN-BNLColumbia-KEK), Phys. Rev. D 68, 054509 (2003).

[2] K. Orginos, T. Blum, and S. Ohta, Phys. Rev. D 73, 094503 (2006).
[3] H.-W. Lin, T. Blum, S. Ohta, S. Sasaki, and T. Yamazaki (RBC Collaboration), Phys. Rev. D 78, 014505 (2008).

[4] T. Yamazaki, Y. Aoki, T. Blum, H. W. Lin, M. F. Lin, S. Ohta, S. Sasaki, R. J. Tweedie, and J. M. Zanotti (RBC and 
UKQCD Collaborations), Phys. Rev. Lett. 100, 171602 (2008).

[5] T. Yamazaki, Y. Aoki, T. Blum, H.-W. Lin, S. Ohta, S. Sasaki, R. Tweedie, and J. Zanotti (RBC and UKQCD Collaborations), Phys. Rev. D 79, 114505 (2009).

[6] Y. Aoki, T. Blum, H.-W. Lin, S. Ohta, S. Sasaki, R. Tweedie, J. Zanotti, and T. Yamazaki, Phys. Rev. D 82, 014501 (2010).

[7] T. Blum, P. Chen, N. H. Christ, C. Cristian, C. Dawson et al., Phys. Rev. D 69, 074502 (2004).

[8] Y. Aoki, T. Blum, N. Christ, C. Dawson, K. Hashimoto et al., Phys. Rev. D 72, 114505 (2005).

[9] C. Allton et al. (RBC and UKQCD Collaborations), Phys. Rev. D 78, 114509 (2008).

[10] Y. Aoki et al. (RBC and UKQCD Collaborations), Phys. Rev. D 83, 074508 (2011).

[11] R. Arthur et al. (RBC and UKQCD Collaborations), Phys. Rev. D 87, 094514 (2013).

[12] T. Blum et al. (RBC and UKQCD Collaborations), Phys. Rev. D 93, 074505 (2016).

[13] G. Martinelli, C. Pittori, C. T. Sachrajda, M. Testa, and A. Vladikas, Nucl. Phys. B445, 81 (1995).

[14] T. Blum et al., Phys. Rev. D 66, 014504 (2002).

[15] Y. Aoki et al., Phys. Rev. D 78, 054510 (2008).

[16] C. Sturm, Y. Aoki, N. Christ, T. Izubuchi, C. Sachrajda, and A. Soni, Phys. Rev. D 80, 014501 (2009).

[17] R. Pohl et al., Nature (London) 466, 213 (2010).

[18] N. Bezginov, T. Valdez, M. Horbatsch, A. Marsman, A. C. Vutha, and E. A. Hessels, Science 365, 1007 (2019).

[19] M. Tanabashi et al. (Particle Data Group), Phys. Rev. D 98, 030001 (2018).

[20] W. Xiong et al., Nature (London) 575, 147 (2019).

[21] T. Bhattacharya, V. Cirigliano, S. Cohen, R. Gupta, H.-W. Lin, and B. Yoon, Phys. Rev. D 94, 054508 (2016).

[22] B. Yoon et al., Phys. Rev. D 95, 074508 (2017).

[23] M. Lin, Proc. Sci. LATTICE2013 (2014) 275 [arXiv: 1401.1476].
[24] S. Ohta (RBC and UKQCD Collaborations), Proc. Sci. LATTICE2013 (2013) 274.

[25] S. Ohta (RBC and UKQCD Collaborations), Proc. Sci. LATTICE2014 (2014) 149.

[26] S. Ohta (RBC, UKQCD, and LHP Collaborations), Proc. Sci. LATTICE2015 (2016) 124.

[27] M. Abramczyk, M. Lin, A. Lytle, and S. Ohta (RBC and UKQCD Collaborations), Proc. Sci. LATTICE2016 (2016) 150.

[28] S. N. Syritsyn et al., Phys. Rev. D 81, 034507 (2010).

[29] C. Alexandrou, S. Gusken, F. Jegerlehner, K. Schilling, and R. Sommer, Nucl. Phys. B414, 815 (1994).

[30] F. Berruto, T. Blum, K. Orginos, and A. Soni, Phys. Rev. D 73, 054509 (2006).

[31] T. Blum, T. Izubuchi, and E. Shintani, Phys. Rev. D 88, 094503 (2013); E. Shintani, R. Arthur, T. Blum, T. Izubuchi, C. Jung, and C. Lehner, Phys. Rev. D 91, 114511 (2015).

[32] J. Dragos, R. Horsley, W. Kamleh, D. B. Leinweber, Y. Nakamura, P. E. L. Rakow, G. Schierholz, R. D. Young, and J. M. Zanotti, Phys. Rev. D 94, 074505 (2016).

[33] J. Liang, Y.-B. Yang, K.-F. Liu, A. Alexandru, T. Draper, and R. S. Sufian, Phys. Rev. D 96, 034519 (2017).

[34] K.-I. Ishikawa, Y. Kuramashi, S. Sasaki, N. Tsukamoto, A. Ukawa, and T. Yamazaki (PACS Collaboration), Phys. Rev. D 98, 074510 (2018).

[35] C. C. Chang et al., Nature (London) 558, 91 (2018).

[36] A. Chodos, R. L. Jaffe, K. Johnson, and C. B. Thorn, Phys. Rev. D 10, 2599 (1974).

[37] G. S. Adkins, C. R. Nappi, and E. Witten, Nucl. Phys. B228, 552 (1983).

[38] M. Gorbahn and S. Jager, Phys. Rev. D 82, 114001 (2010).

[39] L. G. Almeida and C. Sturm, Phys. Rev. D 82, 054017 (2010).

[40] R. Gupta, Y.-C. Jang, B. Yoon, H.-W. Lin, V. Cirigliano, and T. Bhattacharya, Phys. Rev. D 98, 034503 (2018).

[41] T. Harris, G. von Hippel, P. Junnarkar, H. B. Meyer, K. Ottnad, J. Wilhelm, H. Wittig, and L. Wrang, Phys. Rev. D 100, 034513 (2019). 